\title{
Un polarizado Uruguay: tendencias en la estructura agraria 1990 - 2011
}

\author{
A polarized Uruguay: trends in the agrarian structure 1990 - 2011 \\ Um Uruguai polarizado: tendências na estrutura agrária 1990 - 2011 \\ Joaquín Cardeillac ${ }^{1}$ (D) \\ ${ }^{1}$ Núcleo de Estudios Sociales Agrarios (NESA), Departamento de Sociología, Facultad de Ciencias Sociales, Universidad de la \\ República (UdelaR), Montevideo, Uruguay. E-mail: joaquin.cardeillac@gmail.com
}

\begin{abstract}
Cómo citar: Cardeillac, J. (2020). Un polarizado Uruguay: tendencias en la estructura agraria 1990 - 2011. Revista de Economia e Sociologia Rural, 58(4), e210744. https://doi.org/10.1590/1806-9479.2020.210744
\end{abstract}

\begin{abstract}
Resumen: El presente trabajo es una contribución a la comprensión de algunas de las transformaciones que han ocurrido en la sociedad rural del Uruguay contemporáneo. Para hacerlo se procedió a contrastar empíricamente 3 hipótesis. La primera implica estudiar la pertinencia y vigencia de un esquema tradicional acerca de la estructura agraria del Uruguay que distingue entre productores empresariales, productores familiares y asalariados. Para ello se realizó un análisis de componentes principales sobre variables de los Censos Generales Agropecuarios de 1990, 2000 y 2011, y se identificaron conglomerados territoriales distintos según el grado de predominancia de los diferentes tipos de producción (empresarial o familiar). La segunda hipótesis retomada de los antecedentes, propone la existencia de un avance del agronegocio en el agro uruguayo. Para estudiarla, se analizó la distribución de varios atributos identificados por la bibliografía que discute la "lógica" o el "modelo" del agronegocio, para evaluar su penetración en los territorios identificados mediante los análisis de componentes principales y de conglomerados. Por último, una tercera hipótesis sostiene que la lógica del agronegocio colide y es antagónica a la lógica de la producción familiar. El análisis realizado muestra una estructura agraria polarizada, donde los modelos de producción son cada vez más distantes.
\end{abstract}

Palabras clave: estructura agraria, estructura social, agronegocios, producción familiar, producción empresarial.

\begin{abstract}
This paper contributes to the comprehension of transformations that have occurred in the rural society of contemporary Uruguay. To do so, three hypotheses were empirically tested. The first studies the relevance and validity of a traditional scheme on Uruguay's agricultural structure that distinguishes between business producers, family producers, and wage earners. For this purpose, a principal component analysis (PCA) on variables from the General Agricultural Census of 1990, 2000, and 2011 was carried out, and different territorial clusters were identified according to the degree of predominance of different types of production (business or family). The second hypothesis proposes the advance of agribusiness logic in Uruguayan agriculture. To study this, the distribution of several attributes identified by the literature discussing the "logic" or "model" of agribusiness was analyzed, evaluating its incidence in the territories identified by cluster analyses carried out over the factors identified by PCA. Finally, a third hypothesis argues that the logic of agribusiness collides with and is antagonistic to the logic of family production. Therefore, the analysis shows a polarized agrarian structure, where production models are increasingly distant.
\end{abstract}

Keywords: agrarian structure, social structure, agribusiness, family farming, entrepreneurial production.

Resumo: Este artigo contribui para a compreensão das transformações que têm ocorrido na sociedade rural do Uruguai contemporâneo. Para tal, foram testadas empiricamente três hipóteses. A primeira envolve o estudo da relevância e vigência de um esquema tradicional sobre a estrutura agrícola do Uruguai, que distingue entre produtores empresariais, produtores familiares e assalariados. Para tal, foi 
realizada uma análise de componentes principais (ACP) sobre variáveis do Censo Geral Agrícola de 1990, 2000 e 2011, tendo sido identificados diferentes agrupamentos territoriais em função do grau de predominância de diferentes tipos de produção (empresarial ou familiar). A segunda hipótese propõe o avanço da lógica do agronegócio na agricultura uruguaia. Para estudar isto, foi analisada a distribuição de vários atributos identificados pela literatura que discute a "lógica" ou o "modelo" do agronegócio, avaliando sua incidência nos territórios identificados pelas análises de clusters realizadas sobre os fatores identificados pela ACP. Finalmente, uma terceira hipótese argumenta que a lógica do agronegócio colide com e é antagônica à lógica da produção familiar. Em relação a isso, a análise mostra uma estrutura agrária polarizada, na qual os modelos de produção estão cada vez mais distantes.

Palavras-chave: estrutura agrária, estrutura social, agronegócio, agricultura familiar, produção empresarial.

\section{Introducción}

Durante los últimos años, las transformaciones del agro uruguayo han estado en el centro del debate académico y en buena medida, también del debate político. Como suele suceder, las valoraciones acerca de estas transformaciones han sido variadas y en muchos casos opuestas. Una línea de interpretación discute cómo la penetración del capital financiero en el mercado de tierras, generó una valorización inédita de las mismas que concluyó en un proceso de desplazamiento de los productores a favor de Sociedades Anónimas, integradas muchas de ellas por capitales extranjeros (Piñeiro, 2014; Piñeiro, 2010). Retomando esa línea de trabajo, Carámbula (2015) profundiza en los cambios recientes del agro y sus consecuencias teóricas, identificando la consolidación de un patrón de desarrollo novedoso, concentrador y excluyente. Y es que si bien la lógica capitalista es por definición excluyente y concentradora, la intensidad con la que se están excluyendo lógicas alternativas de producción (como la familiar) así como la intensidad de la concentración (en particular de la tierra) que se observa en años recientes, resulta -a su entender- cualitativamente distinta. Según el mismo autor, estos procesos de exclusión y concentración -en perspectiva, recientesatentaron con éxito contra las bases del latifundio ganadero tradicional; es decir, contra lo que ha sido el principio estructurante fundamental del agro uruguayo a lo largo de su historia. Al mismo tiempo, Carámbula (2015) identifica un patrón de desarrollo trasnacional y anónimo, concretado en el creciente control de la tierra productiva uruguaya por parte de actores extranjeros, mediante figuras como las sociedades anónimas. En su lectura, este segundo aspecto resulta particularmente importante, ya que está en la base de un movimiento que intenta diluir las responsabilidades económicas, laborales, ambientales y tributarias del "nuevo" empresariado o "agronegocio" (Carámbula, 2015).

Así pues, desde comienzos del siglo XXI están ocurriendo acontecimientos que revierten el proceso de "estancamiento dinámico" que caracterizó al agro uruguayo hasta el fin del siglo XX (Piñeiro \& Moraes, 2008). Los mismos, impulsaron un crecimiento económico del sector agropecuario inédito y si bien lo más habitual ha sido relacionarlos con el desarrollo de la cadena forestal y la agricultura de secano -específicamente, con la soja-, lo cierto es que también hubo transformaciones e innovaciones en rubros tradicionales, como la ganadería y la lechería (Bervejillo \& Bertamini, 2014).

Con estos procesos como trasfondo, no ha sido poca la atención recibida por las nuevas formas de organización de la producción agropecuaria y del negocio agropecuario en general (Piñeiro \& Cardeillac, 2014; Gras \& Hernández, 2013; Errea et al., 2011, Bisang et al., 2008; Riella \& Romero, 2014). En términos muy básicos el centro de los cambios identificados está en la idea de una "agricultura en red", que a su vez da lugar al surgimiento de un nuevo modelo: los "agro-negocios" (Gras \& Hernández, 2013; Oyhantçabal \& Narbondo, 2008). Tomando estos antecedentes, resulta pertinente estudiar cómo ha cambiado la estructura social rural uruguaya en el período 1990 - 2011. Cabe destacar que si bien existen ejemplos a nivel internacional de trabajos orientados a lograr una aproximación multivariada a la estructura agraria (Wimberley, 1987; Thomas et al., 1996), a nivel nacional no existen antecedentes, por lo que se optó por una estrategia que permitiera avanzar en este aspecto, contribuyendo así también a llenar ese vacío. 


\section{Fundamentos teóricos}

\section{a. Estructura agraria en Uruguay}

En términos muy generales, la estructura social rural del Uruguay se ha considerado estrechamente vinculada a la estructura agraria y ésta última, a su vez, sería básicamente el resultado de la distribución -desigual- de la tierra (Piñeiro \& Moraes, 2008; Fernández, 2002). Siendo así, la sociedad rural del Uruguay se estructuró a partir de tres clases sociales básicas: los productores empresariales (PE), los asalariados rurales y esa "clase incómoda" (Shanin, 1983) que en Uruguay se conoce como Producción Familiar (PF) ${ }^{1}$. De acuerdo a este esquema, los empresarios son propietarios de tierra y la explotan con fines agropecuarios mediante el uso de mano de obra remunerada, quedando el trabajo familiar -cuando lo hay- reservado a las tareas de gerenciamiento. Históricamente, hay una importante tradición de empresas agropecuarias familiares tanto en la ganadería de carne como en la agricultura extensiva, pero también en otros rubros no tradicionales como arroz, citrus, lechería y forestación (Piñeiro \& Moraes, 2008). De acuerdo a Piñeiro \& Moraes (2008) la clase de los empresarios puede subdividirse en dos sub clases: por un lado, los "estancieros" latifundistas, con baja inversión en capital y baja productividad, ubicados hacia el centro y noreste del país y por otro lado, los empresarios agrícola-ganaderos, más propensos a las innovaciones, inversiones en capital (maquinaria y paquetes tecnológicos). Éstos se ubicarían más bien hacia el litoral oeste, hacia el sur y también hacia el este y noreste en el caso de los arroceros (Piñeiro \& Moraes, 2008).

En cuanto a la Producción Familiar (PF), los criterios para delimitar esta clase tienen que ver con la distribución de la tierra, pero no sólo con ella. De hecho se suelen identificar: i) la forma en la que moviliza el trabajo: mayoritariamente por vínculos familiares, y ii) el hecho de que dependen sobre todo de sus propios medios de producción, incluyendo la tierra, más que de la venta de fuerza de trabajo (Oya, 2004). Así la Producción Familiar es una forma de producción destinada al intercambio con potencial para la acumulación privada. Ahora bien, en el caso de la producción familiar, hay una unidad de capital y trabajo (Mann \& Dickinson, 1978). La consecuencia es que, a diferencia de la producción capitalista en la que el trabajo es una mercancía más, en la producción familiar las relaciones de producción son distintas. Partiendo de esa base, se ha insistido mucho en la idea de una superposición entre tres unidades funcionales: i) la unidad de producción (explotación); ii) la unidad de consumo (hogar); y iii) la unidad de parentesco (familia), como característica distintiva de la Producción Familiar. En este caso, el trabajo familiar requerido no es principalmente trabajo gerencial, sino más bien trabajo directo; es decir, trabajo para la generación de bienes primarios (Djurfeldt, 1996).

En el caso de Uruguay esta clase o tipo de productor está presente en todos los rubros, aunque con intensidad variable (Riella, 2010; Rossi, 2010; Piñeiro, 2004; Piñeiro, 1994; Piñeiro, 1991; Piñeiro, 1984; Astori et al., 1982). De modo homólogo a lo que sucede con los empresarios, al interior de estos también es posible construir subclases. En este sentido, la idea de "diferenciación" de la producción familiar justamente alude a la existencia de subtipos de que se distinguen básicamente, en función de su grado de capitalización y posición social (Bernstein, 2009) ${ }^{2}$. Desde el punto de vista geográfico, si bien hay la Producción Familiar está presente en todo el territorio, es clara la existencia de una concentración en los Departamentos del sur, especialmente en Canelones, San José, Lavalleja y Colonia (Piñeiro \& Moraes, 2008).

La tercera clase corresponde a los asalariados rurales o más bien, a los trabajadores y trabajadoras agropecuarias (Cardeillac \& Juncal, 2017). La distinción conceptual resulta

\footnotetext{
${ }^{1}$ La incomodidad aludida por Shanin viene dada por la dificultad que representa la persistencia de esta clase, para los esquemas de clase marxistas más ortodoxos que esperan (por teoría) una polarización entre empresarios y asalariados.

2 En el caso de las lecturas "chayanovianas" esta variabilidad obedecería fundamentalmente a ciclos de vida de la familia (Chayanov, 1974), mientras que en el caso de las lecturas "lenninistas" obedecerían a un proceso de "descomposición" del campesinado y de formación de clase: capitalista (empresario) y proletaria (asalariados) (Lenin, 1972).
} 
pertinente ya que pone el énfasis en la tarea que realizan, así como en el hecho de que reciben por ello un salario, en lugar de poner el énfasis en la residencia (urbana o rural). Ello no incluiría a un gran porcentaje de trabajadores y trabajadoras que residen en las ciudades o centros poblados, al tiempo que sí estaría incluyendo trabajadores con las más diversas tareas, que residen fuera de las ciudades pero no tienen vínculo con el sector agropecuario. En cuanto a la diferenciación interna de esta clase, varios factores distintos han sido indicados en la bibliografía. Uno de ellos es la forma del contrato, temporal o permanente. Otro, el rubro productivo en el que se emplean, que da lugar a tipos como el peón de estancia tradicional ganadero o la trabajadora que cosecha en rubros intensivos. También está el nivel de calificación, así como el lugar de trabajo y residencia (González Sierra, 1994). Además de estas diferenciaciones Carámbula (2009) agrega un elemento más, al explicar que además de los asalariados completamente proletarizados, es muy frecuente encontrar asalariados "semiproletarizados" que al mismo tiempo que venden su fuerza de trabajo en parte del año, siguen trabajando también en su propia explotación de carácter familiar. Desde el punto de vista territorial la nota reciente más relevante tiene que ver con su distribución entre zonas urbanas y rurales ya que mientras en 1985 un $70 \%$ de los asalariados agropecuarios residían en zonas rurales, en 2011 más de un 70\% lo hace en localidades urbanas (Cardeillac \& Nathan, 2015). De todos modos, dado que este artículo se enfoca a discutir la estructura social rural en su vínculo con la estructura agraria, se profundizará bastante menos en esta clase que en las otras dos, para las cuáles las fuentes de datos trabajadas resultan mucho más adecuadas ${ }^{3}$.

\section{b. El modelo o lógica del agronegocio}

Los párrafos anteriores retoman brevemente la discusión acerca de la estructura agraria. No obstante, no son pocos los autores que sugieren de modo más o menos explícito, la insuficiencia de este modelo de tres clases sociales para dar cuenta de la estructura social del agro uruguayo contemporáneo (Piñeiro \& Cardeillac, 2017; Carámbula, 2015). Siendo así, resulta necesario explicitar cuáles son las características distintivas de la "producción en red" (Bisang et al., 2008; Errea et al., 2011; Arbeletche et al., 2012) para poder luego explorar su impacto en la estructura previamente discutida.

Para el caso de la agricultura uruguaya de comienzos del Siglo XXI el contraste entre las formas nuevas y viejas de producir se ha planteado en los términos que siguen (Cuadro1):

Cuadro 1: comparación entre viejos y nuevos productores agrícolas.

\begin{tabular}{|c|c|}
\hline Forma clásica: agricultura tradicional & Nueva forma: agricultura en red \\
\hline $\begin{array}{l}\text { Propietarios de activos: instalaciones, } \\
\text { máquinas, tierra (capital inmovilizado) }\end{array}$ & $\begin{array}{l}\text { Poco o nada de activos en propiedad, arriendo de } \\
\text { tierras (a otros productores que se convierten en } \\
\text { rentistas fundiarios) }\end{array}$ \\
\hline Valor patrimonial & Valor Comercial \\
\hline Baja flexibilidad (largo plazo) & Alta flexibilidad (corto plazo) \\
\hline \multirow[t]{3}{*}{ Mano de obra familiar, asalariada } & $\begin{array}{c}\text { Empleo calificado, asalariados (con participación en } \\
\text { los beneficios), relaciones laborales débiles }\end{array}$ \\
\hline & Innovaciones tecnológicas \\
\hline & Economía de escala (costos de producción) \\
\hline $\begin{array}{l}\text { Lógica productiva: aumentar rendimientos } \\
\text { y volúmenes producidos }\end{array}$ & Lógica financiera: rendimientos del capital \\
\hline
\end{tabular}

\footnotetext{
${ }^{3}$ Los Censos Generales Agropecuarios (CGA) tienen un clarísimo sesgo hacia la información productiva, de hecho, la unidad de análisis de los CGA es el establecimiento agropecuario, es más, sólo aquellos con una superficie superior a 1 hectárea. Siendo así, la información obtenida a partir de análisis sobre los mismos resulta mucho más adecuada para representar a los empresarios y productores familiares (ambos poseen tierra) que a los asalariados agropecuarios.
} 
Cuadro 1: Continuación...

\begin{tabular}{|c|c|}
\hline Forma clásica: agricultura tradicional & Nueva forma: agricultura en red \\
\hline $\begin{array}{l}\text { Gestión del riesgo por diversificación de } \\
\text { producciones }\end{array}$ & $\begin{array}{l}\text { Gestión de riesgos (climático, agronómico, política } \\
\text { fiscal...) por utilización de mercados futuros y por } \\
\text { distribución espacial de la producción nacional e } \\
\text { internacional }\end{array}$ \\
\hline $\begin{array}{c}\text { Poca autonomía comercial (gobernanza } \\
\text { vertical) }\end{array}$ & $\begin{array}{l}\text { Margen de negociación con proveedores y } \\
\text { compradores (gobernanza horizontal) }\end{array}$ \\
\hline $\begin{array}{c}\text { Sumisión a la valorización comercial por el } \\
\text { comprador: normas, calidad, marcas, } \\
\text { diferenciación. }\end{array}$ & $\begin{array}{l}\text { Control y anticipación de la etapa comercial: } \\
\text { volúmenes importantes, calidad masiva. }\end{array}$ \\
\hline Agricultura con agricultores & Agricultura "sin agricultores" \\
\hline Uni-localización & Multi-localización \\
\hline Vive en el medio rural & Residencia urbana o en el extranjero \\
\hline Durabilidad & Movilidad \\
\hline Renta débilmente deslocalizada & Renta fuertemente deslocalizada \\
\hline
\end{tabular}

Fuente: tomado de (Arbeletche et al., 2012: 112)

Por su parte, Gras \& Hernández (2013) resumieron el modelo del agronegocio mediante 10 características: 1 - operan trascendiendo sectores, 2- son cadenas lideradas por compradores globales con un enorme poder de demanda, 3 - implican un uso de capital elevado y en particular, de capital financiero, 4- usan paquetes tecnológicos y transgénia para estandarizar procesos, 5- aumento de escala, 6- acaparamiento de tierras ya sea mediante compra o arriendo, 7 - recurren a pocos asalariados permanentes calificados y muchos temporales no calificados, 8- utilizan y desarrollan de tic's para transacciones globales, 9 - controlan el conocimiento y las innovaciones mediante patentes y 10- separan la propiedad del capital de la dirección del negocio, típicamente recurriendo a la figura de Sociedades Anónimas (Gras \& Hernández, 2013).

Tomando esto como punto de partida y sumado a la discusión sobre la estructura agraria y los distintos tipos de productor, en lo que sigue se procedió a estudiar empíricamente algunas variables para dar cuenta de la estructura del agro uruguayo así como su evolución hacia la incorporación de las características que definen la lógica o modelo del agronegocio.

\section{Metodología}

Para estudiar los cambios en la estructura agraria uruguaya se recurrió a las bases de datos de los tres últimos Censos Generales Agropecuarios (CGA) de 1990, 2000 y 2011. Para poder evaluar la adecuación del modelo de tres clases sociales, se optó por trabajar sobre los únicos agregados territoriales que pueden ser identificados y "seguidos" en los tres censos. Estos agregados se denominan Secciones Policiales (SP) y subdividen a los distintos departamentos del país. La estrategia de análisis consistió en la construcción de variables analíticas que resumen información de las explotaciones o las personas, a nivel de la (SP). Luego, se realizó un análisis de componentes principales (ACP), con la finalidad de resumir la información de las diferentes variables seleccionadas en un número menor de dimensiones capaces de expresar la diversidad de situaciones previstas de acuerdo al marco teórico. Por último y una vez encontrada una solución factorial capaz de dar cuenta de la expresión territorial de la estructura agropecuaria en los tres momentos, se procedió a realizar un análisis de conglomerados que permitiera discriminar conjuntos de SP en los que predominan unos $u$ otros tipos de producción, para finalmente analizar el modo en que distintas características asociadas al modelo del agronegocio se hacen más o menos presentes. 


\section{Descripción de las variables seleccionadas}

Para estudiar la conformación y cambios de la estructura agraria se avanzó en la identificación de factores que permiten distinguir los distintos tipos de producción para estudiar su peso en las SP que distinguen los CGA de 1990, 2000 y 2011. Para eso se construyeron 9 variables que permiten dar cuenta de las diferencias entre tipos de producción empresarial o familiar y entre grados de capitalización dentro de los tipos. Estas variables son:

1. Porcentaje de la superficie de la SP controlada por PF. Corresponde a la suma de hectáreas correspondientes a la superficie total de las explotaciones identificadas como $\mathrm{PF}^{4}$, dividido el total de hectáreas de la SP en la que se encuentran y multiplicado por 100.

2. Porcentaje de la superficie de la SP en mano de Productores Empresariales (PE) ${ }^{5}$ es la suma de hectáreas totales declaradas por Personas Físicas que emplean más de 2 asalariados permanentes al año o más de 500 jornales, dividido el total de hectáreas de la Sección Policial en la que se encuentran y multiplicado por 100.

3. Porcentaje de la superficie de la SP acaparada por Sociedades con Contrato Legal (SCL). Corresponde a la suma de hectáreas totales de las explotaciones cuyo propietario es una persona jurídica (Sociedad Anónima o Sociedad de Responsabilidad Limitada), dividido el total de hectáreas de la SP en la que se encuentran y multiplicado por 100.

4. Porcentaje de las explotaciones que son parte de un conjunto de explotaciones del mismo propietario. Corresponde a la suma de las explotaciones de una SP que corresponden a propietarios que poseen además otras explotaciones, dividido el total de explotaciones de la SP, multiplicado por 100.

5. Porcentaje del trabajo total de la SP que corresponde a trabajo asalariado. Es la suma de trabajadores y trabajadoras asalariadas permanentes sobre el total de trabajadores (asalariados o no asalariados) y multiplicado por 100.

6. Tasa de feminidad. Se trata del cociente entre el número de mujeres de la "población agrícola" de la SP y el número de varones de la misma, multiplicado por 100.

7. Promedio de tractores "nuevos" por explotación en la SP. Se trata del promedio de tractores con 10 años o menos en 1990, o con 5 años o menos en el caso del 2000 y el 2011.

8. Promedio de hectáreas por trabajador. Corresponde al promedio de las hectáreas por trabajador en las explotaciones de la SP. Se calcula la cantidad de hectáreas que hay por trabajador en cada una de las explotaciones de la SP y luego se obtiene el promedio.

9. Promedio de profesionales y técnicos por explotación. Corresponde al promedio de profesionales y técnicos en las explotaciones de la SP7.

A continuación se presenta un resumen de la distribución de las variables para el total de SP en los tres Censos (Tabla 1).

\footnotetext{
${ }^{4}$ En Uruguay, desde 2008 existe una definición oficial de PF. A su vez, de acuerdo a la Resolución ministerial № 1013 de 2016, para que una explotación sea considerada "producción familiar" debe cumplir 4 requisitos: a. Realizar la explotación agropecuaria o actividad productiva agraria con la contratación de mano de obra asalariada de hasta dos asalariados no familiares permanentes o su equivalente en jornales zafrales no familiares de acuerdo con la equivalencia de 250 (doscientos cincuenta) jornales zafrales al año por cada asalariado permanente. b. Realizar la explotación agropecuaria de hasta 500 hectáreas, índice CONEAT 100, bajo cualquier forma de tenencia. c. Residir en la explotación agropecuaria, donde se realice la actividad productiva agraria, o en una localidad ubicada a una distancia no mayor a $50 \mathrm{~km}$. d. Que los ingresos nominales familiares no generados por. la explotación agropecuaria o actividad productiva agraria declarada sean inferiores o iguales a 14 BPC en promedio mensual. Dadas las restricciones de las fuentes de datos, en el caso de este trabajo, para identificar a una explotación como parte de la PF se aplicó el siguiente criterio: explotaciones de personas físicas que controlan como máximo 500 hectáreas y emplean no más de 2 asalariados permanentes o 500 jornales al año. Así, la definición es una elaboración propia pero que retoma los dos criterios centrales de la definición oficial: mano de obra y superficie.

${ }^{5}$ Son Productores Empresariales las personas físicas que emplean más de 2 asalariados permanentes al año o más de 500 jornales.

${ }^{6}$ La población agrícola en los Censos Agropecuarios corresponde a las personas que son residentes habituales (al menos 6 meses al año) de las explotaciones sean o no trabajadores o familiares de los productores.

${ }^{7}$ Este dato no está disponible para 1990.
} 
Tabla 1. Medidas de resumen de las variables seleccionadas: Media y Coeficiente de Variabilidad (CV)

\begin{tabular}{|c|c|c|c|c|c|c|}
\hline \multirow{2}{*}{$\begin{array}{c}\text { Años } \\
\text { Variables }\end{array}$} & \multicolumn{2}{|c|}{1990} & \multicolumn{2}{|c|}{2000} & \multicolumn{2}{|c|}{2011} \\
\hline & Media & CV & Media & CV & Media & CV \\
\hline Promedio de tractores nuevos & 0.24 & 88.92 & 0.08 & 100.10 & 0.14 & 83.30 \\
\hline Promedio de heactáreas por trabajador & 130.56 & 78.57 & 104.80 & 79.04 & 167.58 & 82.72 \\
\hline$\%$ de la superficie de la SP en manos de PF & 28.68 & 69.95 & 29.20 & 69.82 & 27.54 & 77.43 \\
\hline$\%$ de la superficie de la SP en manos de PE & 34.64 & 40.31 & 36.97 & 41.09 & 31.59 & 45.75 \\
\hline$\%$ de la superficie de la SP acaparada por SCL & 18.25 & 69.74 & 21.81 & 66.82 & 32.33 & 52.74 \\
\hline$\%$ del trabajo total que es trabajo asalariado & 44.06 & 45.60 & 37.21 & 43.71 & 50.27 & 37.49 \\
\hline $\begin{array}{l}\% \text { de las explotaciones que son parte de un } \\
\text { conjunto }\end{array}$ & 6.76 & 95.20 & 6.24 & 98.26 & 9.50 & 79.30 \\
\hline Tasa de feminidad (\%) & 46.43 & 27.84 & 65.43 & 24.07 & 47.58 & 29.96 \\
\hline Promedio de profesionales y técnicos & NA & NA & 0.06 & 113.71 & 1.46 & 122.43 \\
\hline
\end{tabular}

Fuente: elaboración propia en base a los microdatos de los censos de 1990, 2000 y 2011.

La propuesta de análisis consiste en probar la adecuación (o inadecuación) de una solución factorial que resuma la información de las 9 variables anteriores para dar cuenta de diferencias entre tipos de producción, que sean consistentes con el modelo conceptual presentado en el repaso de antecedentes, cuya validez se sostenga en los tres momentos. Una vez realizado ese primer paso, se profundizará en el análisis del avance del modelo o lógica de los agronegocios.

\section{Resultados y discusión}

Para resumir la información de las 9 variables anteriores se realizó un ACP. Siguiendo el criterio de Kaiser fue posible identificar, para los tres censos (1990, 2000 y 2011) dos factores que dan cuenta de $70,7 \%$ de la variabilidad total de las variables en $1990,67 \%$ en 2000 y $65,5 \%$ en 2011 y que en todos los años tienen un valor propio superior a la unidad ${ }^{8}$. Luego, para comprender esos componentes principales, se estudiaron las saturaciones factoriales, es decir, el peso de las distintas variables en cada uno de los factores mediante una rotación Varimax, frecuentemente utilizada en ACP. A continuación, se presenta la contribución de cada factor a la variabilidad total para cada uno de los CGA (Tabla 2) y luego la Tabla 3 con las cargas o saturaciones de cada variable en cada factor según el año.

Tabla 2. Varianza total explicada por los componentes en cada año

\begin{tabular}{|c|c|c|c|c|c|c|c|}
\hline & \multirow[b]{3}{*}{ Componente } & \multicolumn{6}{|c|}{ Varianza total explicada } \\
\hline & & \multicolumn{3}{|c|}{ Autovalores iniciales } & \multicolumn{3}{|c|}{$\begin{array}{c}\text { Sumas de rotación de cargas al } \\
\text { cuadrado }\end{array}$} \\
\hline & & Total & $\begin{array}{c}\% \\
\text { varianza }\end{array}$ & $\begin{array}{c}\% \\
\text { acumulado }\end{array}$ & Total & $\begin{array}{c}\% \\
\text { varianza }\end{array}$ & $\begin{array}{c}\% \\
\text { acumulado }\end{array}$ \\
\hline \multirow{2}{*}{ CGA 1990} & 1 & 4.259 & 53.243 & 53.243 & 4.107 & 51.332 & 51.332 \\
\hline & 2 & 1.393 & 17.413 & 70.656 & 1.546 & 19.324 & 70.656 \\
\hline \multirow{2}{*}{ CGA 2000} & 1 & 4.341 & 48.238 & 48.238 & 3.855 & 42.830 & 42.830 \\
\hline & 2 & 1.722 & 19.131 & 67.369 & 2.209 & 24.539 & 67.369 \\
\hline \multirow{2}{*}{ CGA 2011} & 1 & 4.447 & 49.409 & 49.409 & 4.108 & 45.647 & 45.647 \\
\hline & 2 & 1.453 & 16.148 & 65.557 & 1.792 & 19.910 & 65.557 \\
\hline
\end{tabular}

Fuente: elaboración propia en base a los CGA de 1990,2000 y 2011.

\footnotetext{
${ }^{8}$ Los valores del estadístico Kaiser-Meyer-Olkin (KMO) de adecuación muestral son 0,819 en 1990, 0,695 para el 2000
} y 0,736 en el 2011. 
Tabla 3. Cargas factoriales de las distintas variables en los componentes

\begin{tabular}{|c|c|c|c|c|c|c|}
\hline \multirow{2}{*}{ Variable } & \multicolumn{2}{|c|}{1990} & \multicolumn{2}{|c|}{2000} & \multicolumn{2}{|c|}{2011} \\
\hline & Eje 1 & Eje 2 & Eje 1 & Eje 2 & Eje 1 & Eje 2 \\
\hline $\begin{array}{l}\text { Promedio de tractores nuevos en las } \\
\text { explotaciones }\end{array}$ & & 0.87 & & 0.74 & 0.38 & 0.73 \\
\hline Promedio de hectáreas por trabajador & 0.85 & -0.34 & 0.83 & -0.41 & 0.83 & \\
\hline Proporción de la superficie controlada por PF & -0.87 & & -0.90 & & -0.91 & \\
\hline Proporción de la superficie en manos de PE & 0.56 & -0.44 & 0.56 & -0.43 & 0.51 & -0.52 \\
\hline Proporción de la superficie acaparado por SCL & 0.67 & 0.49 & 0.68 & & 0.75 & \\
\hline $\begin{array}{l}\text { Proporción del trabajo total que es trabajo } \\
\text { asalariado }\end{array}$ & 0.86 & & 0.82 & 0.36 & 0.75 & 0.33 \\
\hline Proporción de explotaciones parte de un conjunto & 0.75 & & 0.69 & & 0.80 & \\
\hline Tasa de feminidad en la SP & -0.83 & & -0.83 & & -0.85 & \\
\hline Promedio de profesionales y técnicos & ND & ND & & 0.73 & & 0.55 \\
\hline
\end{tabular}

Fuente: elaboración propia en base a los CGA de 1990, 2000 y 2011.

El primero de los componentes logra dar cuenta de más de un $40 \%$ del total de la varianza en todos los años, llegando incluso a superar el 50\% en el 2000 (Tabla 2). Este primer componente es "bipolar" en el sentido de que resume el efecto de variables con sentidos contrarios (Tabla 3). Lo que captura es la polarización clásica entre dos clases sociales del agro uruguayo: por un lado, los PF y por otro, los PE. A los primeros se asocia la tasa de feminidad de la SP, mientras que a los segundos se asocia la extensividad (promedio de hectáreas por trabajador), el uso de mano de obra asalariada, una incidencia mayor de explotaciones que son parte de un conjunto del mismo dueño y el área en acaparada por Sociedades con Contrato Legal. El segundo factor, en cambio, capta en el entorno de un $20 \%$ adicional de la variabilidad (Tabla 2) y resume básicamente el uso de maquinaria (tractores por explotación), el uso de mano de obra calificada (profesionales y técnicos promedio por explotación) y en menor medida, también el uso de mano de obra asalariada (Tabla 3). Al mismo tiempo, es un factor al que se asocia inversamente la proporción de superficie en manos de PE. La interpretación de esto podría ser menos directa, pero en realidad expresa un hecho bien conocido: la producción agropecuaria empresarial uruguaya se caracteriza por un uso muy extensivo de la tierra y una estrategia de acumulación de riqueza basada en el aumento de superficie para acrecentar las ganancias, más que por una tendencia a la intensificación por medio de la inversión en mano de obra calificada o maquinaria. Así, el componente definido por esas dos variables en un polo (tractores y mano de obra calificada), ubica en el otro a la PE.

Una vez resumida la estructura agraria a partir de esas dos dimensiones recién descritas, se procedió a realizar un análisis de conglomerados (AC) que permitió clasificar a las distintas SP en subconjuntos homogéneos a su interior y heterogéneos entre sí. Estos subconjuntos expresan, básicamente, el predominio de ciertas formas de producción en los distintos territorios. Para todos los Censos fue posible identificar 4 subconjuntos de SP con las siguientes características (Tabla 4):

El primero de los subconjuntos de SP queda conformado por aquellas en las que hay un claro predominio de la producción familiar. A continuación se presenta un cuadro que resume el perfil de este grupo de acuerdo a las variables que se utilizaron en el ACP. 
Tabla 4. Perfil del conglomerado 1 de acuerdo a las variables usadas en el ACP. Años 1990, 2000 y 2011.

\begin{tabular}{cccc} 
Conglomerado 1 & 1990 & $\mathbf{2 0 0 0}$ & $\mathbf{2 0 1 1}$ \\
Número de SP & 72 & 66 & 49 \\
Proporción de la superficie de la SP en manos de PF & 51.2 & 55.0 & 60.0 \\
Tasa de feminidad de la SP 1990 & 58.8 & 82.5 & 66.2 \\
Proporción de la superficie de la SP acaparada por SCL & 8.5 & 10.5 & 14.3 \\
Proporción del trabajo total que es trabajo asalariado & 26.9 & 26.7 & 33.6 \\
Proporción de la superficie de la SP en manos de PE & 22.1 & 21.8 & 14.6 \\
Promedio de heactáreas por trabajador & 37.0 & 25.3 & 25.1 \\
\hline
\end{tabular}

Fuente: elaboración propia en base a los CGS de 1990, 2000 y 2011

Como puede observarse en la Tabla 4, este conjunto de SP ha ido reduciendo su participación en el total. Mientras en 2000 agrupaba 72 SP hacia 2011 apenas agrupa 49. Este aspecto confirma las dificultades que enfrentó la PF a inicios del S XXI.

En cuanto al perfil de este conglomerado, corresponde a SP con fuerte presencia de PF y con una tasa de feminidad muy por encima del promedio y en los que el porcentaje de trabajo que es asalariado en relación al trabajo total, se ubica por debajo del $50 \%$ y muy por debajo del promedio general. Estos aspectos refuerzan la idea de que son SP en las que hay una presencia dominante de formas de producción familiares, en las que se superponen las unidades funcionales de producción, consumo y parentesco (Oya, 2004; Djurfeldt, 1996). Son también territorios en los que la cantidad de hectáreas por trabajador es muy baja -en términos relativos para el caso uruguayo-, demostrando que la PF ha sido y sigue siendo, mucho más intensiva en el uso de mano de obra (familiar o remunerada) que cualquier forma o subtipo de producción empresarial. Por último, estos territorios -dominados por la lógica de producción familiar- se caracterizan también por tener una sub-representación de PE y más específicamente aun, por tener un promedio muy bajo de porcentaje de superficie acaparada por Sociedades con Contrato Legal. Así, aunque hay una tendencia al alza en la participación de personas jurídicas en el control de la tierra, el resultado en estas SP siempre está muy por debajo del promedio general.

El segundo subconjunto de SP tiene un conjunto de características que lo ubica en una situación intermedia (Tabla 5). En este sentido, los territorios agrupados corresponderían a zonas en las que hay tensión entre diferentes tipos de producción. Siendo así, pueden considerarse zonas en transición entre la PF y la PE.

Tabla 5. Perfil del conglomerado 2 de acuerdo a las variables usadas en el ACP. Años 1990, 2000 y 2011.

\begin{tabular}{cccc} 
Conglomerado 2 & $\mathbf{1 9 9 0}$ & $\mathbf{2 0 0 0}$ & $\mathbf{2 0 1 1}$ \\
Número de SP & 80 & 90 & 95 \\
Proporción de la superficie de la SP en manos de PE & 42.0 & 41.8 & 35.1 \\
Proporción de la superficie de la SP en manos de PF & 23.5 & 26.0 & 27.8 \\
\hline
\end{tabular}

Fuente: elaboración propia en base a los CGS de 1990, 2000 y 2011

A diferencia que en el caso del subconjunto anterior, el territorio en situación mixta o en transición ha venido aumentando. Esto resulta claro ya que pasamos de 80 SP de este tipo en 1990, a 90 en 2000 y 95 en 2011 (Tabla 5). La principal característica de este conglomerado de SP está dada por la coexistencia de formas familiares y empresariales de producción 
agropecuaria: en todos los años el porcentaje de superficie en manos de PE supera al promedio del año mientras que el porcentaje de la superficie en manos de PF está siempre muy próxima al porcentaje promedio del año.

Por último, el Análisis de Conglomerados (AC) distinguió dos subconjuntos más (Tabla 6 y Tabla 7). En estos casos, ambos se tratan de SP en los que predomina claramente, la PE. La presentación de sus perfiles permitirá ir captar cuáles son las diferencias entre los dos.

Tabla 6. Perfil del conglomerado 3 de acuerdo a las variables usadas en el ACP. Años 1990, 2000 y 2011.

\begin{tabular}{cccc} 
Conglomerado 3 & $\mathbf{1 9 9 0}$ & $\mathbf{2 0 0 0}$ & $\mathbf{2 0 1 1}$ \\
Número de SP & 30 & 30 & 38 \\
Promedio de tractores nuevos por explotación & 0.6 & 0.2 & 0.3 \\
Proporción de la superficie acaparada por SCL & 32.0 & 36.1 & 50.0 \\
Proporción del trabajo total que es trabajo asalariado & 59.6 & 55.0 & 68.4 \\
Proporción de explotaciones parte de un conjunto & 7.1 & 7.6 & 11.1 \\
Promedio de técnicos y profesionales & ND & 0.2 & 3.0 \\
Proporción de la superficie de la SP en manos de PE & 30.1 & 37.7 & 28.8 \\
\hline
\end{tabular}

Fuente: elaboración propia en base a los CGS de 1990, 2000 y 2011

Tabla 7. Perfil del conglomerado 4 de acuerdo a las variables usadas en el ACP. Años 1990, 2000 y 2011.

\begin{tabular}{cccc} 
Conglomerado 4 & 1990 & $\mathbf{2 0 0 0}$ & $\mathbf{2 0 1 1}$ \\
Número de SP & 50 & 47 & 57 \\
Promedio de heactáreas por trabajador & 268.3 & 226.1 & 331.4 \\
Proporción del trabajo total que es trabajo asalariado & 68.6 & 53.4 & 63.2 \\
Proporción de explotaciones parte de un conjunto & 14.4 & 13.9 & 19.0 \\
Proporción de la superficie de la SP acaparada por SCL & 28.8 & 31.0 & 41.3 \\
Proporción de la superficie de la SP en manos de PE & 43.6 & 48.4 & 42.2 \\
\hline
\end{tabular}

Fuente: elaboración propia en base a los CGS de 1990, 2000 y 2011

Retomando lo discutido en la fundamentación conceptual, es bastante claro que estos dos conglomerados agrupan SP con predominio de uno u otro de los dos tipos de productores empresariales identificados en los antecedentes. Así, mientras el conjunto de SP del tercer conglomerado se caracteriza por tener un grado de inversión en maquinaria mayor, así como un uso también superior de mano de obra técnica o profesional (Tabla 6); el cuarto se caracteriza más bien por tener un promedio de hectáreas por trabajador muy elevado y una frecuencia muy superior de establecimientos que son parte de un conjunto de un mismo dueño (Tabla 7). Mientras el conglomerado 3 (Tabla 6) agrupa a SP dominadas por productores empresariales que basan su estrategia de acumulación y ganancia en la productividad; en el 4 (Tabla 7) se concentran más bien SP con dominio de empresarios que basan su acumulación y ganancia en aumentar su superficie y que dependen menos de maquinaria. Por último, es conveniente resaltar que tanto en el conglomerado 3 como en el 4 hay una importante participación de las Sociedades con Contrato Legal en el control de la tierra, de hecho, tanto en el Conglomerado 4 como en el Conglomerado 3 el porcentaje de la 
superficie total de las SP acaparado por SCL está siempre (para todos los años) muy por encima del promedio general.

Con la finalidad de lograr una evaluación más acabada de lo que podríamos denominar "hipótesis de inadecuación" -es decir, la idea de que el esquema tradicional de clases ya no es adecuado- a partir de los conglomerados construidos, se presenta a continuación un conjunto de mapas con su distribución en el territorio (Figura 1).

De acuerdo a esa distribución varios aspectos resultan claros. En primer lugar, la ubicación de los distintos tipos de SP en función del predominio de un tipo u otro de producción, sigue un patrón consistente con los antecedentes y el "modelo tradicional" (Piñeiro \& Moraes, 2008). Claramente las SP con más predominio de PF se ubican al sur, aunque aparecen también SP de menor tamaño en los alrededores de las ciudades capitales (Cluster 1). Luego hay un tipo empresarial más del tipo del estanciero y latifundista que domina en las SP del centro y norte del país (Cluster 4), y un tipo empresarial agrícolaganadero, que invierte en capital y domina en SP ubicadas hacia el Litoral, hacia el Sur y también en algunas del Este, vinculadas al arroz y la forestación (Cluster 3) (Figura 1). Dicho de otro modo, los resultados del ACP y AC realizados sobre los CGA confirman el esquema tradicional: es posible construir un gradiente producción familiar / empresarial y otro relativo al estilo empresarial, que se confirma en los tres censos y que también en 1990, 2000 y 2011, identifica 4 conglomerados cuyas características entre censos se parecen mucho más que entre conglomerados dentro de cada censo.
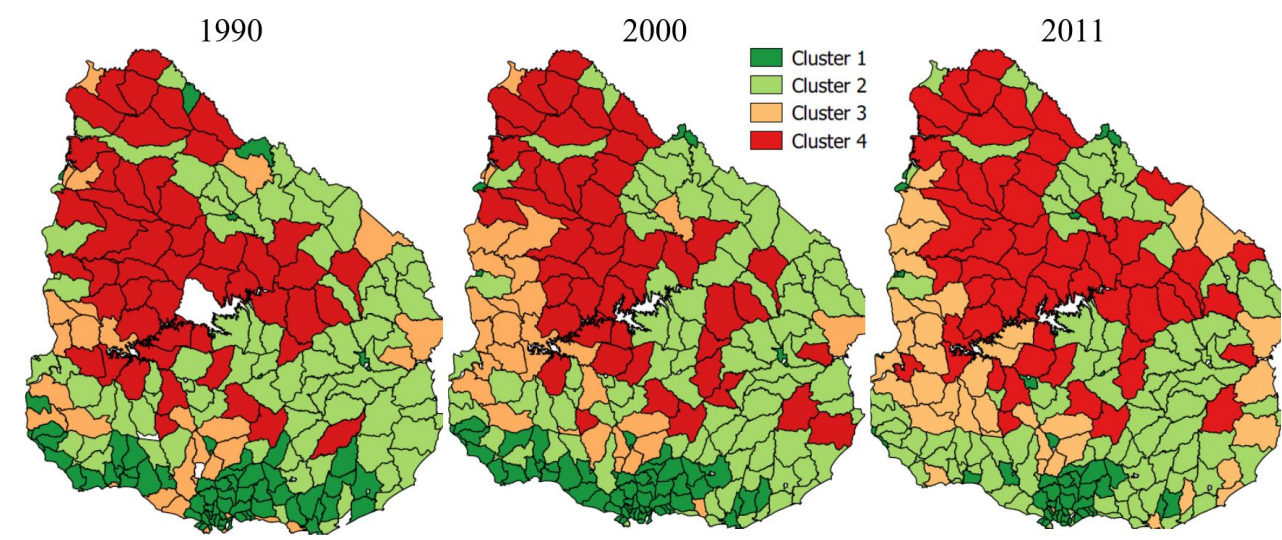

Figura 1: distribución en el territorio de los conglomerados de SP, para 1990, 2000 y 2011.

En este sentido, la evidencia falsea la "hipótesis de inadecuación" e indica que no hay prisa para dejar de lado el esquema tradicional de clases que se ha utilizado para conceptualizar el campo uruguayo y que abreva de una tradición intelectual muy potente (Chayanov, 1974; Shanin, 1983). De hecho, la aplicación del mismo resulta adecuada para visualizar algunos de los cambios más relevantes del período. En ese sentido, mientras en el período 1990 - 2000 la tendencia fue a un aumento de las zonas mixtas o transicionales, en las que coexistían PE y PF sin que dominara más una que otra, junto a un aumento también del área con dominio de empresarios "agrícola-ganaderos"; el período 2000 - 2011 se caracteriza por un avance de todas las formas de producción empresarial y una fuerte contracción de la producción familiar.

\section{La lógica del agronegocio}

Un último aspecto a explorar es el grado de avance de la lógica o modelo del agronegocio. De acuerdo a los antecedentes, más que un nuevo tipo de actor, el agronegocio es un nuevo tipo de lógica (Gras \& Hernández, 2013). Siguiendo con ese razonamiento, su penetración no implica desplazamiento de un tipo de actor por otro, sino que debería observarse una tendencia al alza en ciertas características que han sido propuestas como definitorias de esta nueva lógica, para el caso de los diferentes territorios delimitados en el 
análisis anterior. La idea, es utilizar el esquema de análisis propuesto antes para probar un conjunto de hipótesis manejadas por la bibliografía y que pueden considerarse sucesivamente. En este sentido, se propone partir de la expresión territorial de la estructura agraria obtenida mediante el ACP y AC realizados, que permitió distinguir entre 4 tipos de territorios. Uno dominado por la producción familiar, que se ha contraído y vuelto más homogéneo entre 1990 y 2011 . Otro, de transición o híbrido, en el que pesan tanto la PF como la PE y que ha aumentado su participación, especialmente entre 1990 y 2000 . Un tercer territorio dominado por un empresario típicamente capitalista "agrícola-ganadero", que invierte en maquinaria y mano de obra calificada. Y un cuarto tipo de territorio básicamente dominado por un empresariado latifundista-estanciero, centrado en la tierra como medio de acumulación. La lógica es analizar y comparar la evolución de indicadores de agronegocios en esos 4 tipos de territorios (Tabla 8), a fin de contrastar empíricamente dos hipótesis adicionales:

1- El agronegocio es un modelo o lógica y no un tipo de actor, por lo tanto, su avance sobre las formas empresariales del agro uruguayo implica que las variables utilizadas como indicadores de agronegocios tendrán una variación al alza en los territorios con dominio de producción empresarial (Clusters 3 y 4).

2- El modelo del agronegocio se opone al modelo de la producción familiar. En consecuencia, se observará una tendencia a la polarización entre los territorios de la PFy los de la PE, especialmente en lo que hace a las variables utilizadas como indicadores de agronegocios.

Para que el razonamiento anterior se sostenga, es condición que las variables a estudiar no sean parte de las utilizadas en la generación de los factores (y luego Clusters). Si bien algunas de esas variables y su evolución tienen indudablemente que ver con la penetración del modelo del agronogocio -es claro, por ejemplo, el peso creciente de las SCL en el control de la tierra- sería improcedente analizar su asociación con los conglomerados en tanto que ya forman parte de la construcción de los mismos. El procedimiento seguido, en cambio, propone lo siguiente: si la conceptualización acerca de los agronegocios como modelo es acertada, debería observarse un aumento en indicadores asociados al agronegocio en los territorios dominados por los distintos tipos de productores empresariales. A su vez, si la conceptualización de que el agronogocio es un modelo que enfrenta a la producción familiar (Piñeiro \& Cardeillac, 2014; Cardeillac, 2015) es acertada, debería además observarse una tendencia distinta en esos mismos indicadores, que polarice los territorios de la PF y los de los PE.

Los datos que siguen (Tabla 8), presentan la evolución de algunas características asociadas al agronegocio y disponibles en la información censal para el período 2000 - 2011. Se seleccionaron 3 variables adicionales. Por un lado, la presencia promedio de administradores al frente de explotaciones de las SP. De acuerdo a los antecedentes, una característica del agronegocio es la separación entre la propiedad del capital y la dirección del negocio. En este sentido, el promedio de administradores -en lugar de productores- al frente de explotaciones de la SP, resulta un buen indicador de la separación de la propiedad del capital y la dirección del negocio (Gras \& Hernández, 2013; Arbeletche et al., 2012). Otra de las variables seleccionadas es el promedio de hectáreas en arrendamiento de la SP. También siguiendo a los antecedentes, el arrendamiento es una forma de controlar tierra típica del agronegocio, por lo que es esperable que el promedio de hectáreas en arrendamiento sea superior en las SP dónde la penetración del agronegocio es mayor. Y por último, se estudió también la evolución de la superficie promedio de las explotaciones, en el entendido de que el aumento de escala, es otra de las características indicadas por la bibliografía antecedente como típica de la lógica de agronegocio. 
Tabla 8. Indicadores de la lógica del agronegocio, evolución 2000 - 2011 según conglomerados

\begin{tabular}{|c|c|c|c|c|c|c|}
\hline & \multicolumn{2}{|c|}{$\begin{array}{c}\text { Promedio de } \\
\text { administradores }\end{array}$} & \multicolumn{2}{|c|}{$\begin{array}{c}\text { Promedio de hectáreas } \\
\text { en arrendamiento }\end{array}$} & \multicolumn{2}{|c|}{$\begin{array}{c}\text { Tamaño promedio de las } \\
\text { explotaciones }\end{array}$} \\
\hline & 2000 & 2011 & 2000 & 2011 & 2000 & 2011 \\
\hline Cluster 1 & 16,1 & 9,5 & 4.723 & 2.201 & 66 & 51 \\
\hline Cluster 2 & 24,6 & 22,2 & 19.131 & 15.412 & 302 & 294 \\
\hline Cluster 3 & 26,5 & 32,3 & 19.086 & 27.891 & 590 & 605 \\
\hline Cluster 4 & 29,6 & 39,3 & 26.558 & 29.307 & 828 & 913 \\
\hline
\end{tabular}

Fuente: elaboración propia en base a los CGA de 2000 y 2011.

Los resultados obtenidos al analizar la tendencia de las variables seleccionadas como indicadores de la lógica del agronegocio (Tabla 8), resultan bastante contundentes en dos aspectos. Por un lado, se detecta con claridad una intensidad creciente en los fenómenos relevados por estas variables en las SP con dominio de producción empresarial (ya sea ésta latifundista-estanciera o capitalista "agrícola-ganadera"). Y por otro lado, se observa también un creciente distanciamiento entre los tipos básicos de producción. Comenzaremos por analizar el primero de los movimientos.

En cuanto a la separación del gerenciamiento y la propiedad, es posible observar que el promedio de administradores en las SP con dominio de la producción empresarial (Clusters 3 y 4 ) aumenta entre el 2000 y el 2011, pasando de 26,5 a 32,3 y de 29,6 a 39,3 respectivamente. Esta tendencia confirma la hipótesis relativa a una imposición creciente de la lógica del agronegocio entre los empresarios del agro, más que la idea de un desplazamiento de un tipo de actor por otro. Dicho de otro modo, los resultados en esta variable confirman que en los territorios dominados por cualquiera de los tipos de producción empresarial, se extiende la lógica de la separación de la propiedad del capital y la dirección del negocio. Por un lado están los dueños (ya sea bajo la forma de personas físicas o jurídicas) y por otro los gerenciadores, manejando el establecimiento con la finalidad de obtener ganancias. Adicionalmente, la tendencia al alza en este indicador de la lógica del agronegocio se observa tanto entre los productores empresariales estanciero-latifundistas -que se orientan a obtener renta de la tierra- como en los capitalistas "agrícola-ganaderos" -guiados básicamente por la ganancia obtenida por aumentos en la productividad-. Así, la clasificación en esos dos subtipos, resulta al menos parcialmente cuestionada: si bien es cierto que es posible aun distinguir entre los dos subconjuntos de empresarios, parece claro también que al menos algunas características atribuidas al empresariado latifundista-estanciero no se sostienen. En particular, no parece existir una aversión generalizada a la innovación (Piñeiro \& Moraes, 2008), ya que de hecho, han incorporado sin dificultades la lógica de separar propiedad y gerenciamiento mediante el uso de figuras jurídicas. En consecuencia, debería especificarse que la resistencia a la innovación tiene que ver con la inadecuación de ciertas innovaciones al objetivo principal: la captación de renta. Luego, se debe evitar confundir ese comportamiento con algún tipo de carácter atrasado o tradicionalista: ni bien han estado disponibles innovaciones organizativas adecuadas a ese objetivo, el empresariado estanciero-latifundista las ha adoptado con intensidad.

Otro indicador utilizado es el promedio de hectáreas en arrendamiento en las SP. En general, hay un consenso bastante amplio de que esta forma de controlar tierras es típica de la "producción en red" o los "agronegocios" (Piñeiro \& Cardeillac, 2014; Gras \& Hernández, 2013; Arbeletche et al., 2012). En base a los resultados es posible observar nuevamente, un aumento en el número promedio de hectáreas en arrendamiento en las SP de los dos conglomerados con dominio de formas empresariales de producción. En consecuencia, la evidencia construida confirma la tendencia del indicador anterior para el caso de los territorios de la producción empresarial. En el caso de las SP del Cluster 3 (PE capitalista "agrícola-ganadera") el número de hectáreas arrendadas promedio pasó de 19.086 a 27.891, 
mientras que en el caso de las SP correspondientes al Cluster 4 (PE "estanciero-latifundista") ese promedio pasó de 26.558 ha a 29.307 ha.

En el caso de este indicador es necesario tener presente que su adecuación para captar la "lógica del agronegocio" resulta mayor para el caso de la agricultura, que para otras cadenas de valor. Así, el modelo de organización en red en el que la empresa productora tiene "poco o nada de activos en propiedad" funcionó bien al inicio en el caso de la agricultura, y no tanto en otras cadenas. Un caso paradigmático es la cadena forestal, en la que se observa una integración vertical con coordinación de tipo jerárquica (Sturgeon, 2008). Allí las empresas procesadoras han invertido fuertemente en activos y en particular en tierras, que representan una reserva de valor, además de un medio productivo (Lagaxio, 2013). Por otro lado, también se ha observado que las estrategias de los actores van variando. Un ejemplo de esto es el cambio que observan Gras \& Hernández (2016) cuando mencionan que algunas de las empresas más exitosas del modelo "sin tierra" del agronegocio y la agricultura en red, pasan más adelante a hacer enormes inversiones en tierra en propiedad, como respuesta y adaptación a las inversiones de capitales internacionales que recibieron y que por definición exigen atender con particular énfasis el problema de los costos de transacción, -asegurar el riesgo de la inversión con activos físicos (Gras \& Hernández, 2016). En suma, si bien el indicador se comporta del modo esperado por hipótesis (aumentando en las SP de los conglomerados de producción empresarial) también permite constatar una especificación: el recurso al arrendamiento se da con mayor intensidad en los territorios de la producción empresarial capitalista "agrícola-ganadera" orientada a la ganancia por productividad, que en los territorios de la producción empresarial "latifundista-estanciera" orientada a la captación de rentas.

El último indicador seleccionado es la superficie promedio de las explotaciones de las SP. En este caso los resultados son menos contundentes, pero siguen también el sentido esperado. Así, en el caso de los territorios con domino de producción empresarial "latifundista-estanciera" es posible registrar un aumento marcado de la superficie promedio de las explotaciones que pasan de 826,6 ha a 913 ha. Por su parte, en el caso de los territorios con domino de producción empresarial "agrícola-ganadera" el aumento aunque existe es moderado, pasando de 590 ha a 605 ha.

En resumen, podemos afirmar que los resultados comentados confirman la hipótesis de un avance de la lógica del agronegocio en los territorios de la Producción Empresarial, pero ¿qué sucede en el resto?

De acuerdo con los antecedentes, la lógica del agronegocio sería básicamente antagónica a la de la producción familiar (Piñeiro \& Cardeillac, 2017; Carámbula, 2015; Piñeiro \& Cardeillac, 2014; Cardeillac, 2015). Siendo así, se planteó la hipótesis de que su penetración genera un distanciamiento adicional entre los territorios de la producción empresarial y los de la familiar. Los resultados obtenidos también confirman esta hipótesis. Si se analizan las tendencias de los tres indicadores seleccionados, es posible observar que la situación de los territorios con dominio de la Producción Familiar y Empresarial en 2011 es más distante, de lo que era en el 2000. De hecho, mientras los indicadores aumentan en las SP de la PE, en el caso de las SP con dominio de la PF se observan consistentemente disminuciones: hay menos administradores (el promedio pasa de 16 en 2000 a 9,5 en 2011), menos hectáreas en arrendamiento (el número de ha en arrendamiento de las SP pasa de 4.723 en 2000 a 2.201 en 2011) y menor escala promedio (pasan de 66 ha promedio en 2000 a 51 ha en 2011).

\section{Conclusiones}

En este artículo nos planteamos realizar una descripción analítica, ordenada y sistemática, de algunos cambios relevantes en la estructura agraria del Uruguay. Para lograr ese objetivo, optamos por organizar el trabajo en base a tres hipótesis que se derivan de los antecedentes que se han propuesto para conceptualizar la realidad del campo uruguayo a comienzos del SXXI. La primera hipótesis, sostiene que los esquemas de clase tradicionales han perdido su capacidad para captar la estructura agraria y social del Uruguay rural. Con la finalidad de contrastar esta hipótesis se procedió a estudiar la adecuación de una solución factorial que resumiera en pocas dimensiones la estructura agraria. Ese estudio consistió en 
seleccionar 9 variables consistentes con los antecedentes conceptuales para posteriormente ajustar un modelo que lograra un resumen estable en el tiempo (1990, 2000 y 2011). Los resultados obtenidos en este aspecto refutan la "hipótesis de inadecuación" del esquema tradicional de tres clases, en la medida en que logra identificar con éxito territorios con características que expresan las diferencias entre los tipos de producción de modo consistente con los esperado de acuerdo al esquema de clases tradicional y particularmente, de acuerdo a lo esperado según el clivaje básico entre producción familiar y empresarial ${ }^{9}$.

Una vez realizado ese primer análisis, se procedió a estudiar la segunda hipótesis. En este caso, se optó por trabajar sobre la idea de que el agronegocio debe ser comprendido como una lógica o modelo, más que como un nuevo tipo de actor que desplaza a otro. Siendo así, sus características definitorias deberían manifestarse con particular intensidad entre los productores empresariales en 2011. A este respecto los resultados son muy contundentes y muestran un claro avance de los indicadores de agronegocios en todos los territorios con dominio de formas empresariales de producción para el período 2000 - 2011. Un elemento adicional que se observa, es la tendencia a asimilarse de los territorios de ambos subtipos de producción empresarial. En este sentido, la tendencia observada es a una penetración de la lógica del agronegocio entre los dos tipos de producción empresarial, que ubica a los dos subtipos en una tendencia común y crecientemente divergente de la producción familiar ${ }^{10}$. Y esta es justamente la tercera hipótesis explorada: la lógica del agronegocio como una lógica antagónica a la lógica de la producción familiar. En este aspecto, si bien el tipo de información analizada no es la más adecuada para estudiar conflictos, si es posible aproximarse a esta noción de antagonismo mediante un análisis del distanciamiento creciente. En este aspecto el análisis confirma la existencia de tendencias divergentes entre los tipos de producción empresarial y familiar, que cada vez resultan ser más distintas, al tiempo que pone en evidencia el fuerte proceso de contracción territorial de la lógica de la producción familiar frente al avance de las lógicas empresariales de la mano del agronegocio.

En síntesis, los resultados presentados en este trabajo contribuyen a sistematizar mediante evidencia empírica y de modo original, algunas de las transformaciones más relevantes del agro uruguayo a inicios del SXXI. De entre ellas, una de las más evidentes tiene que ver con la contracción de la producción familiar frente a la empresarial y otra tiene que ver con la penetración del modelo del agronegocio en la producción empresarial. Como resultado de ambas se produce una clara polarización de la estructura social asociada a la estructura agraria, aunque al mismo tiempo, el agro esté cada vez más hegemonizado por un modelo productivo y de negocios centrado en la generación de ganancias. En ese sentido, no hubo ni hay un solo Uruguay en el campo, como tampoco hay un sólo modelo o camino al desarrollo. No obstante, dada la contracción de las formas de producción familiar, y el avance del modelo del agronegocio, no resulta claro que esto vaya a seguir siendo así indefinidamente y por tanto, se hace necesario revisar cual se elige impulsar.

\section{Bibliografía}

Arbeletche, P., Coppola, M., \& Paladino, C. (2012). Análisis del agro-negocio como forma de gestión empresarial en América del Sur: el caso uruguayo. Agrociencia, 16(2) 110-119.

Astori, D., Pérez Arrarte, C., Goyetche, L., \& Alonso, J. (1982). La agricultura familiar uruguaya: orígenes y situación actual. Montevideo: FCU.

\footnotetext{
9 Podría argumentarse que sí confirman parcialmente cierta inadecuación del esquema tradicional en la medida en que los subtipos de producción empresarial son menos diferentes entre sí hacia 2011 de lo que lo eran previamente. Esto debería ser profundizado en otros análisis orientados a contrastar una hipótesis de "inadecuación parcial".

10 Como bien nos hizo ver un revisor del trabajo, es importante recordar que este análisis se realiza a nivel de agregados territoriales y no de explotaciones. En ese sentido, es necesario evitar la falacia de nivel o falacia ecológica: la afirmación de que los territorios de la PF cada vez se distancian más de los empresariales en cuanto a las características del agronegocio, no debe ni puede trasladarse directamente a nivel de las explotaciones. Así, bien puede ser que un cierto subconjunto de explotaciones de la PF haya incorporado elementos típicos de la lógica del agronegocio aun cuando a nivel del territorio la distancia aumente. Como es claro, ese análisis queda fuera del alcance de este artículo.
} 
Bernstein, H. (2009). V.I. Lenin and A.V. Chayanov: looking back, looking forward. The Journal of Peasant Studies, 36(1), 55-81.

Bervejillo, J. E., \& Bertamini, F. (2014). Cambio técnico y crecimiento de la productividad total del sector agropecuario. In OPYPA, Anuario 2014. Montevideo: MGAP.

Bisang, R., Anlló, G., \& Campi, M. (2008). Una revolución (no tan) silenciosa. Claves para repensar el agro en Argentina. Desarrollo Economico

Carámbula, M. (2009). Tiempos de Ausencia. Movilidad espacial y precariedad laboral en los trabajadores rurales temporales: el caso de los esquiladores de Villa Sara. Montevideo: Letraeñe Ediciones.

Carámbula, M. (2015). Imágenes del campo uruguayo en clave de metamorfosis. Cuando las bases estructurales se terminan quebrando. Revista de Ciencias Sociales, 28(36) 17-36.

Cardeillac, J. (2015). Agronegocios y sociedad rural: una relación difícil. Revista de Ciencias Sociales, 28(36), 9-16.

Cardeillac, J., \& Nathan, M. (2015). Caracterización sociodemográfica de la situación de los colectivos de trabajadores rurales y domésticos en el período 1996-2011. In F. Pucci, D. Piñeiro, A. Juncal, \& S. Nión, Sindicalización y negociación en los sectores rural y doméstico (pp. 55 - 81). Montevideo: Mundo Gráfico.

Cardeillac, J., \& Juncal, A. (2017). Estructura agraria y trabajo en un contexto de cambio: el caso de Uruguay. Mundo Agrario, 18, 1-13.

Chayanov, A. V. (1974). La organización de la unidad económica campesina. Buenos Aires: Nueva Visión.

Djurfeldt, G. (1996). Defining and operationalizing family farming from a sociological perspective: European Society for Rural Sociology. Sociologia Ruralis, 36(3)

Errea, E., Peyrou, J., Secco, J., \& Souto, G. (2011). Transformaciones en el agro uruguayo: Nuevas instituciones y modelos de organización empresarial. Montevideo: Universidad Católica del Uruguay.

Fernández, T. (2002). La estructura agraria en el Uruguay entre 1951 y el 2000: Una aproximación descriptiva desde la distribución de la tierra. Centro de Estudios Sociológicos, 20(59), 387-424.

González Sierra, Y. (1994). Los olvidados de la tierra: Vida, organización y luchas de los sindicatos rurales. Montevideo: Nordan-Comunidad.

Gras, C., \& Hernández, V. (2013). Los pilares del modelo agribusiness y sus estilos empresariales. In C. Gras, \& V. Hernández. El agro como negocio: Producción, sociedad y territorios en la globalización (365 p.). Buenos Aires: Biblos.

Gras, C., \& Hernández, V. (2016). Radiografía del nuevo campo argentino: Del terrateniente al empresario trasnacional. Buenos Aires: Siglo Veintiuno.

Lagaxio, L. (2013). La cadena global de valor foresto industrial. Montevideo: DS-FCS.

Lenin, V. I. (1972). El desarrollo del capitalismo en Rusia. Santiago de Chile: Empresa Editora Nacional Quimantu LTDA.

Mann, S., \& Dickinson, J. M. (1978). Obstacles to the development of a capitalist agriculture. The Journal of Peasant Studies, 5(4), 466-481.

Oya, C. (2004). The empirical investigation of rural class formation: Methodological Issues in a Study of Large and Mid-Scale Farmers in Senegal. Historical Materialism, 12(4), 289-326.

Oyhantçabal, G., \& Narbondo, I. (2008). Radiografía del agonegocio sojero. Montevideo: Tradinco S.A.

Piñeiro, D. (1984). Formas de Resistencia de la Agricultura Familiar: el caso del N.E. de Canelones. Montevideo: Editorial Banda Oriental.

Piñeiro, D. (1991). La Agricultura Familiar: El fin de una época. In D. Piñeiro. Nuevos y No Tanto: Los Actores Sociales para la Modernización del Agro Uruguayo. Montevideo: CIESU. Ed. Banda Oriental.

Piñeiro, D. (1994). Tipos Sociales Agrarios y Racionalidad Productiva: un ensayo de interpretación. Montevideo: Dep. de Ciencias Sociales. Facultad de Agronomía.

Piñeiro, D. (2004). El capital social en la producción familiar. Ciclo de conferencias aportes para el futuro de la granja, 40 Años de Inia Las Brujas. Montevideo: INIA.

Piñeiro, D. (2010). Concentración y extranjerización de la tierra en el Uruguay. In M. Manzanal \& G. Neiman. Las agriculturas familiares del MERCOSUR: Trayectorias, amenazas y desafíos (pp. 153-170). Buenos Aires: CICCUS.

Piñeiro, D. (2014). Asalto a la tierra: el capital financiero descubre el campo uruguayo. Capitulo V. In G. Almeyra, L. Concheiro Bórquez, J. M. Mendes Pereira \& C. W. Porto-Gonçalves. Capitalismo: tierra y 
poder en América Latina (1982-2012) Argentina, Brasil, Chile, Paraguay, Uruguay. (Vol. 1). Buenos Aires: Ediciones Continente.

Piñeiro, D., \& Cardeillac, J. (2014). Producción familiar y agronegocios: dos modelos en conflicto. ALASRU Asociación Latinoamericana de Sociología Rural, 10, 187-205.

Piñeiro, D., \& Cardeillac, J. (2017). The Frente Amplio and agrarian policy in Uruguay. Journal of Agrarian Change, 17, 365-380.

Piñeiro, D., \& Moraes, M. I. (2008). Los cambios en la sociedad rural durante el siglo XX. In B. Nahum. El Uruguay del siglo XX: La Sociedad (Vol. 3; pp. 105-136). Montevideo: Banda Oriental.

Riella, A. (2010). Producción familiar y cambios en la estructura social agraria del Uruguay. In M. Manzanal \& G. Neiman. Las agriculturas familiares del mercosur: Trayectorias amenazas y desafíos (pp. 243-263). Buenos Aires: CICCUS.

Riella, A., \& Romero, J. (2014). Continuidades y rupturas en la estructura agraria en el Uruguay del siglo XXI. Pampa (Santa Fe), 10(10), 159-171.

Rossi, V. (2010). La producción familiar en la cuestión agraria uruguaya. Revista Nera, 13(16), 63-82.

Shanin, T. (1983). La clase incómoda. Sociología política del campesinado en una sociedad en desarrollo. (Rusia 1910 - 1925). Madrid: Alianza Editorial.

Sturgeon, T. J. (2008). From Commodity Chains to Value Chains: Interdisciplinary theory building in an age of globalization. Massachusetts: MIT.

Thomas, J., Howell, F., Wang, G., \& Albrecht, D. (1996). Visualizing trends in the structure of U.S. Agriculture, 1982-1992. Rural Sociology, 61, 349-374.

Wimberley, D. (1987). Dimensions of U.S. Agriestructure: 1969-1982. Rural Sociology, 52, 445-461.

Submetido: 6/Ago./2018

Aceito: 27/Abr./2019

JEL: Q15, 013, P16 